\title{
The Radiation Dose Measurement System for the BaBar Electromagnetic Calorimeter
}

\author{
Akram Khan, W. T. Meyer, Joerg Stelzer, Jong Yi
}

\begin{abstract}
An array of 116 p-channel radiation sensitive MOSFET transistors (RadFETs) has been operational for the past 6 years at the BaBar experiment at the PEP-II asymmetric BFactory at the Stanford Linear Accelerator Center (SLAC). This system maps the integrated dose absorbed by different regions of the Electromagnetic Calorimeter (EMC) during the running of the experiment. We report on the design and implementation of the system and finally, the performance of the monitoring system during the last 6 years of BaBar data-taking.
\end{abstract}

\section{INTRODUCTION}

B ABAR is a major particle physics experiment [1] taking data since 1999 at the Stanford Linear Accelerator Center (SLAC), producing many billions of events. The BaBar detector consists of five subdetectors, of which Electromagnetic Calorimeter (EMC) is a key detector for measuring the energies of photons and electrons [2]. EMC is comprised of 6580 of 32$\mathrm{cm}$ long crystals of thallium-doped cesium iodide that scintillate when ionizing radiation traverses them. The response of the crystals, however, can be degraded by accumulated exposure to ionizing radiation. One of the major challenges of the experiment is to operate in a high radiation environment and to monitor the accumulated exposed radiation. To accomplish this, 116 RadFETS have been distributed throughout the detector, of which 56 are at the front faces of barrel crystals, and 60 are at the front faces of the endcap crystals, where we expect to receive the largest dose.

The details of the RadFETs and its monitoring system have been previously described [3], and we only summarize the major parts of the system in the following sections. The emphasis of this paper is on the new version of the monitoring board and the discussion of the performance of the system in the last six years of the operation.

\section{OVERVIEW OF RADFET OPERATION}

RadFET devices are p-type field effect transistors with a specially-grown thick gate oxide layer which has deep wells

Manuscript received November 11, 2005. This work was supported in part by the U.S. Department of Energy, division of High Energy Physics and by PPARC and the British Council, UK.

A. Khan is with the School of Engineering and Design, Brunel University, Uxbridge, UK (e-mail: akram@slac.stanford.edu).

W. T. Meyer is with the Department of Physics and Astronomy, Iowa State University, Ames, IA, USA (e-mail: meyer@iastate.edu).

J. Stelzer is with the Department of Physics, Stanford University, Stanford, CA, USA (e-mail: stelzer@slac.stanford.edu).

J. Yi is with the School of Physics and Astronomy, University of Manchester, Manchester, UK (e-mail: jong@slac.stanford.edu). to trap the positive charge from electron-positron pairs created by ionizing radiation traversing the device. The negative charge is not trapped and must be removed from the device to ensure proper operation. As the gate oxide layer accumulates charge, the voltage between the source and the drain when a fixed current flows through the device increases. Monitoring this source-drain voltage difference is how the integrated radiation is determined.

The RadFET Monitoring Board (RMB) provides a selectable constant current (160 $\mu A$ or $90 \mu A$, with $160 \mu A$ being preferred) and digitizes the resulting voltage levels from up to 32 RadFETs. At all times other than during readout, all three RadFET leads are tied to ground. During readout, the gate and drain remain at ground and the current is fed into the source. A simplified diagram of this is shown in Figure 1. A block diagram of the RMB is shown in Figure 2.

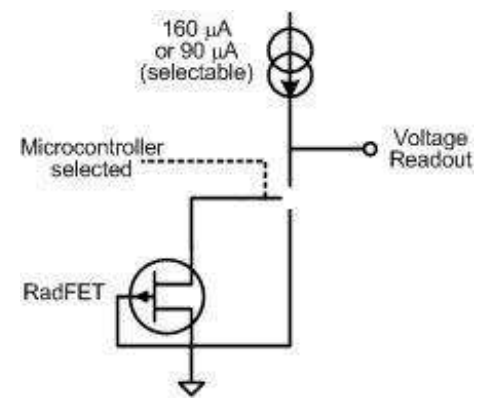

Fig. 1. The readout principle of the RadFETs

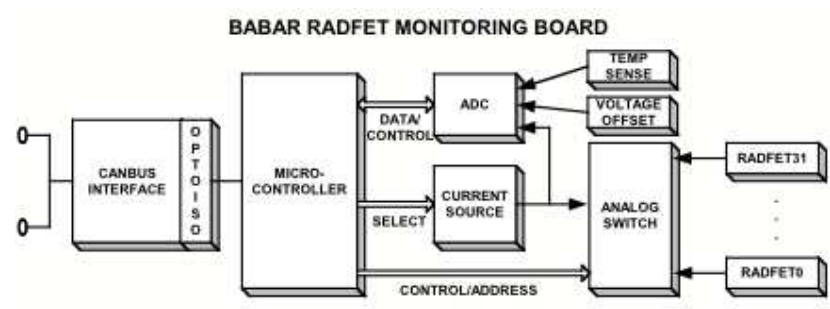

Fig. 2. A block diagram of the RadFET Monitoring Board

In order to achieve a larger range of operation at high resolution, we subtract an offset voltage from the reading before digitization. This offset is a common value for all 32 RadFETs read by a single RMB. The ADC has an input range from $0 \mathrm{~V}$ to $4 \mathrm{~V}$; the RadFET at zero exposure had a readout value of around seven to eight volts. The offset can be adjusted up to 
$10.24 \mathrm{~V}$. Thus, the maximum unscaled value the RMB can read is $14.24 \mathrm{~V}$.

We expect, during the lifetime of the experiment, a voltage shift of 4.5 to $5.0 \mathrm{~V}$. While we are in no immediate danger of going beyond the range of the $\mathrm{ADC}$, this means that sometime during the lifetime of $\mathrm{BaBar}$, we will need to remove the present cards and adjust the offset voltage. Recall also that 32 RadFETs share a common offset and that the natural variation of the starting readout voltages was on the order of a volt. This reduces the range we can tolerate before an offset adjustment to approximately $3.0 \mathrm{~V}$.

This situation, along with a rare mechanical problem of the crystal oscillator, which provides the clock for the microcontroller on the RMB, being torn off the board with its metal casing under the shear force of the magnet field, led to the development of the second generation of $\mathrm{RMB}$

\section{RMB VERSION 2}

Addressing the issues discussed above, the second version of the RMB was proposed and installed for operation in 2002. The improvements to the original version are described below, and the photo of the second version is shown in Figure 3.

- Remote adjustment of the offset voltage. By using a digital resistor that can be adjusted by CANbus commands to the microcontroller, the need for manually adjusting the offset voltage was eliminated.

- Digital resistor divider to scale down large readout voltages. Should the readout voltage exceed the highest level with subtraction $(14.24 \mathrm{~V})$, the readout will be scaled down. This, of course, reduces the resolution by the same factor.

- Replacement of the metal oscillator. An oscillator without a metal case replaced the old type, making the board resistant to the large magnetic field.

- Addition of ability to read the raw voltage from the RadFETs. By using digital resistors, the raw unsubtracted voltage could optionally read out. This would not be as precise, as the reading needs to be scaled to fit the $4 \mathrm{~V}$ range but would allow a reading even if the offset voltage circuit fails. It adds reliability to the readout.

- Addition of resistors to hold the RadFET bias at ground when the RMB is powered off. The original design relied on an active component, the input analog switch, to connect the RadFET source to ground when it was not being read. If the RMB power was turned off, the RadFET was no longer properly grounded. The amount of charge deposited was very small - pico Coulombs per day - but over time could build up to the point where it could affect the calibration of the RadFET. To prevent this, a $10 \mathrm{M} \Omega$ resistor between each RadFET source lead and ground was put in. This value was sufficient to drain the small amount of charge, but will not noticeably affect the value during readout.

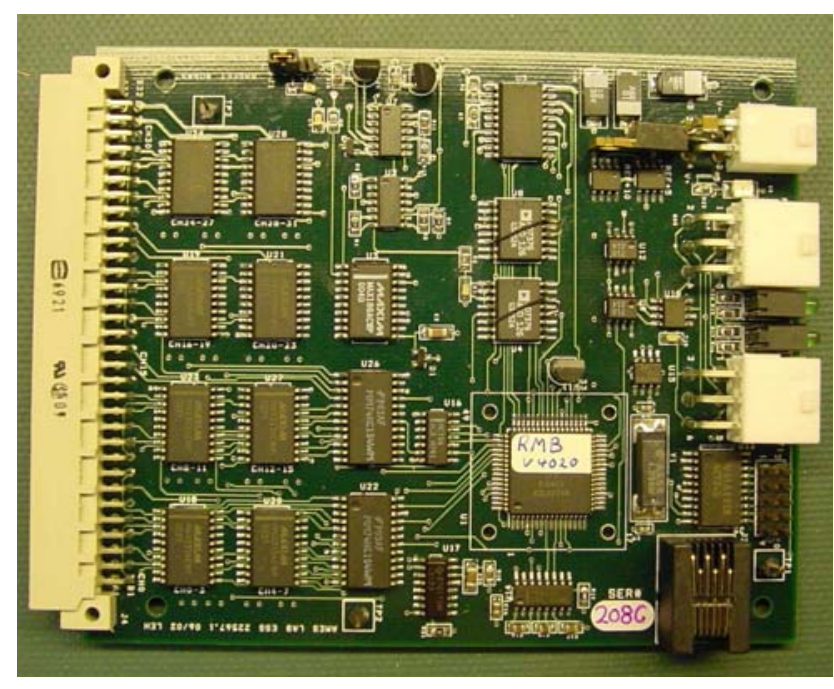

Fig. 3. A photo of the second generation RadFET Monitoring Board

\section{PERFORMANCE}

BaBar saw its first beam in June 1999. Since the beginning, there have been five running periods. Figure 4 shows the daily and integrated luminosity delivered to the $\mathrm{BaBar}$ detector since the start of the experiment.

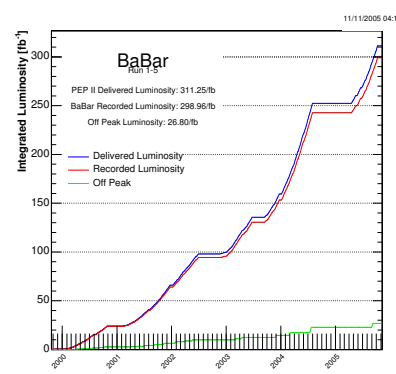

(a)

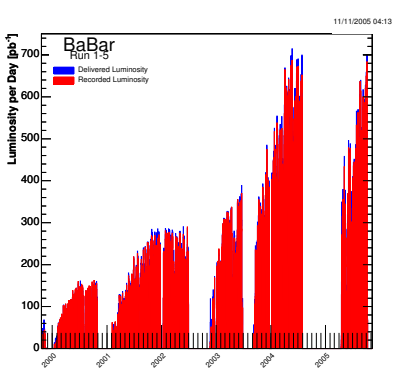

(b)
Fig. 4. (a) The integrated luminosity delivered to the BaBar detector as a function of time. (b) The daily delivered luminosity to the BaBar detector as a function of time.

The RadFETs have been operational the entire time, and the plots of the averaged measured dose as a function of time for different regions of the calorimeter is shown in Figures 5 and 6. Typically, the individual RadFETs will vary by as much as a factor of two from the average, depending on their location in the calorimeter.

As expected, the endcap received the largest dose [4]. The forward half of the barrel received slightly more than the backward half, and this was also expected due to the asymmetric energies of the beams. Also, west half of the detector received slight more dose than the east half; this was expected as well, as the west side is more affected by the higher energy beam. One feature of note is the evidence for annealing in the RadFETs during the shutdown periods: 4 months at the end of 2000, 5 months at the end of 2002, 2 months in the middle of 2003 and 8 months at the end of 2004. This is due to the effects of 


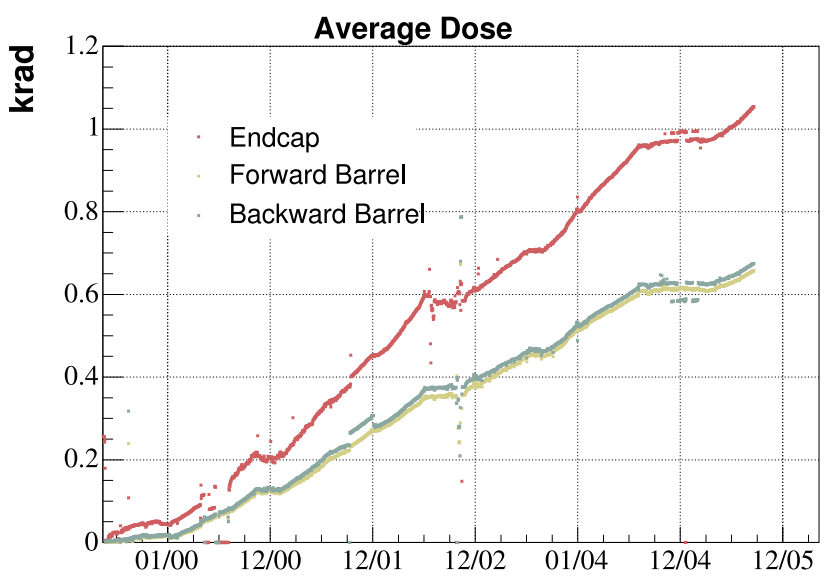

Fig. 5. The temperature corrected average dose seen by the RadFETs in the three regions of the detector. As expected, the endcap receives the largest dose and the forward half of the barrel receives a larger dose than the backward half.

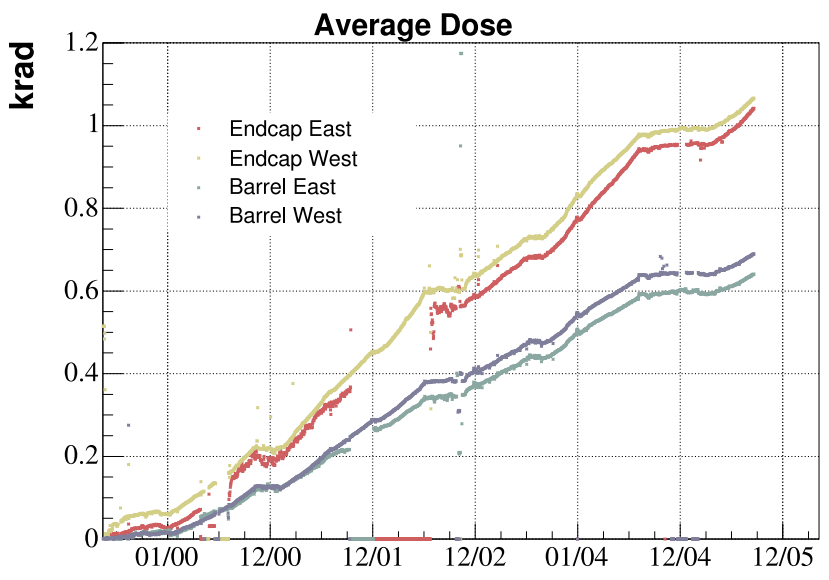

Fig. 6. The temperature corrected average dose seen by the RadFETs in the four regions of the detector. As expected, west side of the detector receives a larger dose.

anti-fading followed by fading as described in section II and the magnitude of the effect is consistent with observations of fading made in [5] and references therein. The data have not been corrected for this effect. Also visible in all figures are the gaps in the data due to a mechanical failure of RMB described above before the second generation of the RMBs were put into operation in 2002; the RadFETs continued to integrate during these gaps, even though we were unable to read them.

Finally, Figures 7 and 8 show the average dose in the different regions of the detector as a function of luminosity delivered. While one should expect a rough correlation between the luminosity and the observed dose, it will not be a linear relationship for several reasons. First, when there is a startup after a long shutdown, there is a period of accelerator tuning which produces high backgrounds in the detector. Second, as data taking progresses the accelerator operators refine their tunes so

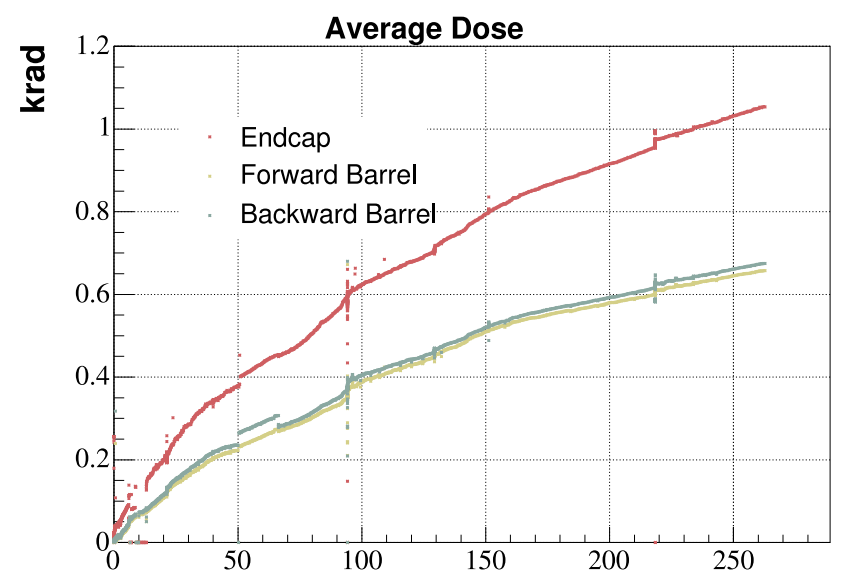

Fig. 7. The average dose in the three regions of the detector versus the integrated luminosity.

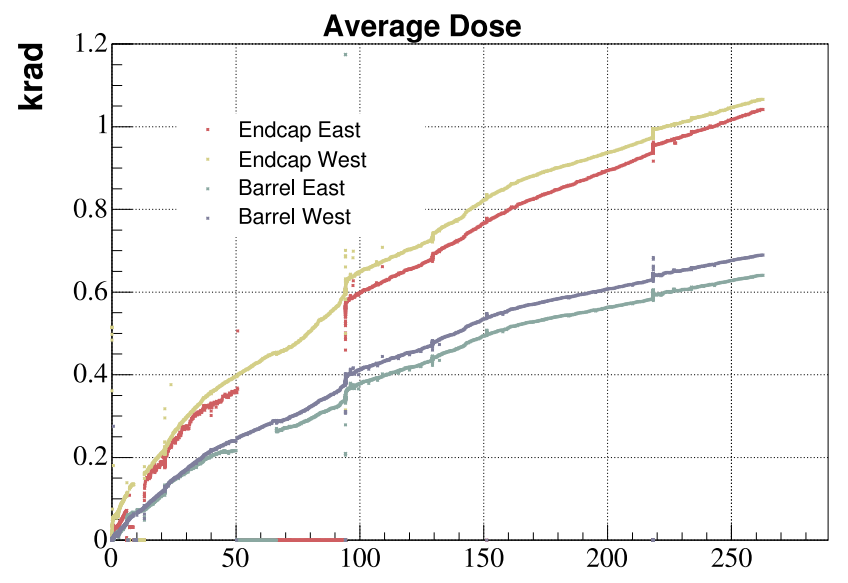

Fig. 8. The average dose in the four regions of the detector versus the integrated luminosity.

that backgrounds decrease. The major source of radiation seen by the RadFETs is beam background, especially while beams are being injected, not while the beams are colliding for physics events.

\section{CONCLUSiON}

The RadFET sensors and the RadFET Monitoring Boards for observing the integrated radiation dose seen by the BaBar Electromagnetic Calorimeter have been operational during all data-taking periods. The average accumulated dose in the endcap region is $1.1 \mathrm{kRad}$ while it is $0.7 \mathrm{kRad}$ for the barrel region. They are well within the budgeted amount based on the expected lifetime of the detector. This system is the largest array of integrating MOS dosimeters to be assembled to date and the most advanced form of automated monitoring used on such devices. These and future readings form an important part of the corrections to be applied to Electromagnetic Calorimeter data. 


\section{ACKNOWLEDGMENT}

The authors thank H.B. Crawley, R.L. McKay and E.I. Rosenberg for their efforts in designing and building the second generation of the RMB and everyone in the BaBar EMC group for all their contributions to the calorimeter. They congratulate and extend their gratitude to the whole BaBar Collaboration and the PEP-II accelerator group for their tremendous accomplishments.

\section{REFERENCES}

[1] BaBar Collaboration, "The BaBar Detector," B. Aubert et al., Nucl. Instr. Meth., Vol. A476, 1 (2002).

[2] J. Bauer, "The BaBar Electromagnetic Calorimeter: Status and Performance Improvements," this conference record.

[3] B. Camanzi et al., "The BaBar RadFET Monitoring Board", IEEE Trans. Nucl. Sci., Vol. 49 Issue 3, pp. 1275-1280 (2002).

[4] A. Hasan, "Backgrounds in the forward region of the BaBar Electromagnetic Calorimeter," BaBar Note 381, SLAC, Stanford, CA, 1997.

[5] N. G. Blamires et. al., IEEE Trans. Nucl. Sci., NS-33(6) pp. 1310-1317 (1986) 